\title{
High frequency of simultaneous presence of ESBL and carbapenemase producers among nosocomial coliform isolates in Faisalabad, Pakistan
}

\author{
Sofia Irfan ${ }^{1}$, Aysha Azhar², Asad Bashir ${ }^{3}$, \\ Salman Ahmed ${ }^{4}$, Abdul Haque ${ }^{5}$
}

\begin{abstract}
Objectives: The objective of the current study was to find prevalence of relevant ESBL and carbapenemase producing genes in nosocomial $E$. coli and $K$. pneumoniae isolates and to check phenotypic susceptibility of all ESBL positive isolates to carbapenems.

Methods: Forty ESBL producing clinical isolates of Escherichia coli $(n=33)$ and Klebsiella pneumoniae $(n=7)$ were examined for the presence of B-lactamase genes (CTX-M, CTX-M-1, 2, 3, 4 and TEM). Carbapenem resistance was checked phenotypically and by presence of blaNDM-1 gene.

Results: Nine (27\%) were positive for CTX-M genes, and 10 (30\%) for TEM among E. coli isolates. Importantly, six isolates showed co-existence of CTX-M and TEM genes. In $K$. pneumoniae, two (28\%) isolates were positive for CTX-M and one (14\%) for TEM genes. Eight (24\%) E. coli and one (14\%) K. pneumoniae isolates were positive for CTX-M-1. Respective figures for CTX-M-4 were three (10\%) and one (14\%). CTX-M-2 and CTX-M-3 groups were not represented. Twenty $(50 \%)$ isolates were resistant to both imipenem and meropenem out of which only four isolates expressed blaNDM-1 gene.

Conclusions: The significant presence of both ESBL and carbapenemase producers and co-existence of ESBL and carbapenemases in the same isolates is worrisome.
\end{abstract}

KEYWORDS: E.coli; K. pneumoniae, Extended Spectrum beta-lactamase (ESBL), Carbapenemase.

Abbreviations: ESBL: Extended spectrum B-lactamase. MBL: Metallo-betlactamase. PCR: Polymerase chain reaction.

doi: https://doi.org/10.12669/pjms.37.1.3192

How to cite this:

Irfan S, Azhar A, Bashir A, Ahmed S, Haque A. High frequency of simultaneous presence of ESBL and carbapenemase producers among nosocomial coliform isolates in Faisalabad, Pakistan. Pak J Med Sci. 2021;37(1):34-39. doi: https://doi.org/10.12669/pjms.37.1.3192

This is an Open Access article distributed under the terms of the Creative Commons Attribution License (http://creativecommons.org/licenses/by/3.0), which permits unrestricted use, distribution, and reproduction in any medium, provided the original work is properly cited.

1. Sofia Irfan, B.Sc. (Hons.).

2. Aysha Azhar, PhD.

Postgraduate Research Laboratory,

The University of Faisalabad, Faisalabad, Pakistan.

3. Asad Bashir, MPhil.

4. Salman Ahmed, B.Sc. (Hons.).

5. Abdul Haque, PhD.

1,3-5: Department of Health Biotechnology,

Akhuwat FIRST, Faisalabad, Pakistan.

Correspondence:

Prof. Dr. Abdul Haque,

Director,

Akhuwat FIRST,

Faisalabad, Pakistan.

Email: ahaqnibge@gmail.com

* Received for Publication:

* Revision Received:

* Revision Accepted:
July 3, 2020

August 20, 2020

August 25, 2020

\section{INTRODUCTION}

Enterobacteriaceae are one of the main causes of bacterial infections in the world. In this family, Escherichia coli and Klebsiella spp. are the most prevalent among causes of nosocomial infections. A broad spectrum of clinical infections are caused by these pathogens in both immunocmpetent and immunocompromised patients. They are also among major causes of nosocomial infections. ${ }^{1}$

One of the most important therapeutic choices for treating infections in both humans and animals is $\beta$-lactam antibiotics. The increase in bacterial resistance to these antibiotics over the past twenty 
years is due to selective pressure caused by use and misuse of these antibiotics. The most relevant mechanism of resistance is the production of $\beta$-lactamases, which hydrolyze the $\beta$--lactam ring of these antibiotics. ${ }^{2}$ Point mutations produce variants which produce the so-called extended spectrum $\beta$-lactamases (ESBLs) responsible for extensive drug resistance.

After detection of the first pathogen that was found expressing ESBL in Germany in $1983,{ }^{3}$ it was defined as $\beta$-lactamases capable of hydrolysing third-generation cephalosporins and aztreonam, but inhibited by clavulanic acid.

Resistance to $\beta$-lactams mediated by (ESBL) is especially relevant in Enterobacteriaceae. ${ }^{4}$ In recent years, these enzymes have acquired a high capability of dissemination, especially in clinical environments. Beside the initially described TEM and SHV ESBLs, more recently CTX-M, OXA, and PER $\beta$-lactamases have been reported. ${ }^{5}$

Infections caused by ESBL producing E. coli and Klebsiella spp are often treated by carbapenems. Among carbapenems, imipenem (IPM) and meropenem (MEM) have been most extensively used. However, resistance towards these drugs has started to emerge due to the production of carbapenamases that degrade all $\beta$-lactam antimicrobials. ${ }^{6}$

One of the major modes of action of these enzymes is to promote membrane impermeability which usually results in hydrolyzation of all penicillins, cephalosporins, and carbapenems. Mutations or alterations in porin channels may also cause membrane impermeability resulting in porin non-functionality or may be due to complete loss of the OmpF and/or OmpC porin proteins. Active drug efflux may also play a role. ${ }^{7}$

The emergence of carbapenem resistance has aggravated the situations as carbapenems are considered the last line of defense against these pathogens. It is the need of the hour to create awareness and device better control measures to halt emergence of drug resistance among Enterobacteriaceae throughout the world. ${ }^{8}$

The main objective of the current study was to explore the phenotypic spectrum of ESBL and carbapenemase producing E. coli and $K$. pneumoniae among isolates from a local nosocomial source using disc diffusion method and to identify relevant genes in an effort to help control measures. This study has specifically addressed ESBL and carbapenemase related resistance towards $\beta$-lactam antimicrobials which were once considered the effective antimicrobials with good safety profiles. Resistance mechanisms for other antimicrobial groups were not included in this study. Another limitation is that it is related only to hospital infections.

\section{METHODS}

Collection of specimens: The bacterial isolates investigated in this study were taken from human blood, urine, sputum, pus, throat, and ear samples collected at Madinah Teaching Hospital (MTH) Laboratory, The University of Faisalabad, Faisalabad, Pakistan from September 2019 to February 2020. These samples were obtained from all age groups irrespective of the gender of the patients. After initial screening for ESBL productions by phenotypic test, the ESBL producing isolates were transported to Akhuwat Faisalabad Institute of Research Science and Technology (Akhuwat FIRST), Faisalabad, Pakistan.

Biochemical Identification of $E$. coli and $K$. pneumonia: Biochemical identification of isolates was done by MR (Methyl Red) and VP (VogesProskauer) tests according to manufacturers' instructions (Merck, Germany).

Phenotypic detection of ESBL producers by double disc synergy method: An isolate showing enhanced zone of $\geq 5 \mathrm{~mm}$ with cefotaxime combined with clavulanic acid as compared to a zone of antibiotic alone by disc diffusion method was phenotypically designated as ESBL producer. ${ }^{9}$

Molecular confirmation of E. coli: PCR was used to confirm $E$. coli isolates by targeting a housekeeping gene uidA that encodes for ß-glucuronidase with primers uidA-F ATC ACC GTG GTG ACG CAT GTC GC and uidA-R CAC CAC GAT GCC ATG TTC ATC TGC..$^{10}$ Each $100 \mu \mathrm{L}$ reaction mixture comprised of $1 \times$ PCR buffer (50 $\mathrm{mM} \mathrm{KCl}, 10 \mathrm{mM}$ Tris $\mathrm{HCl}$; $\mathrm{pH} 8.3$ ); $2.5 \mathrm{mM} \mathrm{MgCl}_{2}$; dNTP's $0.2 \mathrm{mM}$ each; $50 \mathrm{pmol}$ of each primer; 5 $\mathrm{U}$ of recombinant Taq polymerase (Fermentas) and 20ng of DNA template. The thermal cycler conditions were: denaturation for five minutes at $94^{\circ} \mathrm{C}$; 30 cycles of amplification at $94^{\circ} \mathrm{C}$ for one minute, $50^{\circ} \mathrm{C}$ for one minute and $72^{\circ} \mathrm{C}$ for one minute; and finally extension at $72^{\circ} \mathrm{C}$ for seven minutes. The PCR products were visualized by electrophoresis on $2 \%(\mathrm{w} / \mathrm{v})$ agarose gel.

Antimicrobial susceptibility testing: E. coli and $K$. pneumoniae isolates were tested for their susceptibility to carbapenems; imipenem (IPM), and meropenem (MEM) by disc diffusion method. The 
results were interpreted following the guidelines of National Committee for Clinical Laboratory Standards (NCCLS, 2015). ${ }^{11}$

Molecular identification of antimicrobial drug resistance genes: PCR was used to detect CTX-M, TEM, CTX-M-1, CTX-M-2, CTX-M-3, CTX-M-4 and NDM-1 genes. The primer sequences and amplicon sizes are summarized in Table-I.

DNA amplification was performed in a thermal cycler. Each $100 \mu \mathrm{L}$ reaction mixture contained 10X PCR buffer $(50 \mathrm{mM} \mathrm{KCl}$, 10mMTris $\mathrm{HCl}$; $\mathrm{pH} 8.3$ ); $2.5 \mathrm{mM} \mathrm{MgCl}$; dNTPs $0.2 \mathrm{mM}$ each; 50 pmol of each primer; $5 \mathrm{U}$ of recombinant Taq polymerase (Fermentas) and 20ng of DNA template. PCR conditions for the blaCTX-M, blaTEM, blaCTX-M-1, blaCTX-M-2, blaCTX-M-3 and blaCTX-M-4 genes comprised an initial denaturation step for 5 minutes at $95^{\circ} \mathrm{C}$, followed by 30 cycles at $95^{\circ} \mathrm{C}$ for 1 minute, $45^{\circ} \mathrm{C}$ for 30 seconds and $72^{\circ} \mathrm{C}$ for one minute, with a final extension step at $72^{\circ} \mathrm{C}$ for 10 minutes. For blaNDM-1, the amplification cycle consisted of five minutes at $95^{\circ} \mathrm{C}$, followed by 30 cycles at $94^{\circ} \mathrm{C}$ for 1 minute, $55^{\circ} \mathrm{C}$ for 1 minute and $72^{\circ} \mathrm{C}$ for one minute, with extension at $72^{\circ} \mathrm{C}$ for seven minutes. The PCR products were visualized by electrophoresis on $1.5 \%(\mathrm{w} / \mathrm{v})$ agarose gel.

Ethical Committee Approval: The study was approved by Research Committee (Ref: AKTFST/ Misc/2020-104, Dated: 16-07-2020) of Akhuwat FIRST, Faisalabad, Pakistan.

\section{RESULTS}

Isolation and Biochemical Identification of Isolates: A total of 40 ESBL producing isolates showed rose pink, mucoid and non-mucoid colonies indicative of coliform bacteria. The biochemical tests by MR-VP identified 33 isolates as E. coli $(82.5 \%)$ and 7 isolates as K. pneumonia $(17.5 \%)$.

PCR for Confirmation of E.coli Isolates: PCR confirmed the presence of uidA gene in 33 isolates.

Antimicrobial Drug Susceptibility Testing by Disc Diffusion: Of 33 ESBL producing E. coli,18 (54.54\%), and $16(48.48 \%)$ were resistant to imipenem and meropenem respectively and $14 / 33(42 \%)$ isolates were resistant to both of the carbapenems. Four out of seven $(57.14 \%)$ Klebsiella pneumoniae were resistant to both Imipenem and Meropenem (Table-II).

Molecular Identification of ESBL and Carbapenemase producing genes:

Presence of CTX-M and TEM genes was observed in $13(39 \%)$ isolates of $E$. coli and two $(28.5 \%)$ isolates of $K$. pneumoniae (Table-III). Three (9\%) isolates were positive for CTX-M gene alone, four (12\%) for TEM gene alone and six (18\%) showed amplification for both CTX-M and TEM genes in E. coli. So nine (27\%) isolates were CTX-M and 10 $(30 \%)$ were TEM positive among E. coli isolates. In case of K. pneumoniae, one isolate was positive for both CTX-M and TEM genes and one isolate

Table-I: Primer sequences for PCR of drug resistance genes in ESBL producers

\begin{tabular}{llll}
\hline Gene & Primers & Oligonucleotide Sequences & Amplicon Size (bp) \\
\hline \multirow{2}{*}{ blaCTX-M } & CTX-M-F & SCSATGTGCAGYACCAGTAA & $585^{21}$ \\
& CTX-M-R & ACCAGAAYVAGCGGBGC & \\
blaTEM & TEM-F & ATGAGTATTCAACATTTCCG & $867^{22}$ \\
& TEM- $R$ & CTGACAGTTACCAATGCTTA & \\
blaCTX-M-1 & CTX-M-1-F & GACGATGTCACTGGCTGAGC & $499^{15}$ \\
& CTX-M-1-R & AGCCGCCGACGCTAATACA & \\
blaCTX-M-2 & CTX-M-2-F & GCGACCTGGTTAACTACAATCC & $351^{15}$ \\
& CTX-M-2-R & CGGTAGTATTGCCCTTAAGCC & \\
blaCTX-M-3 & CTX-M-3-F & CGCTTTGCCATGTGCAGCACC & $307^{15}$ \\
& CTX-M-3-R & GCTCAGTACGATCGAGCC & \\
blaCTXM-4 & CTX-M-4-F & GCTGGAGAAAAGCAGCGGAG & $474^{15}$ \\
& CTX-M-4-R & GTAAGCTGACGCAACGTCTG & \\
blaNDM-1 & NDM-1-F & GGGCAGTCGCTTCCAACGGT & $475^{23}$ \\
\hline
\end{tabular}


Sofia Irfan et al.

Table-II: Antimicrobial susceptibility pattern of test isolates.

\begin{tabular}{lcccc}
\hline $\begin{array}{l}\text { Antibiotics } \\
(10 \mu \mathrm{g})\end{array}$ & $\begin{array}{c}\text { Susceptibility } \\
\text { Escherichia coli }\end{array}=33$ & $\begin{array}{c}\text { Susceptibility } \\
\text { Klebsiella pneumoniae n=7 }\end{array}$ \\
\hline Imipenem(IPM) & Susceptible & Resistant & Susceptible & Resistant \\
Meropenem (MEM) & 15 & 18 & 3 & 4 \\
\hline
\end{tabular}

Table-III: Distribution of relevant genes in ESBL and CP producing Isolates.

\begin{tabular}{llccc}
\hline Sl. No & Gene Name & E. coli $(n=33)$ & K. pneumoniae $(n=7)$ & Total $(n=40)$ \\
\hline 1 & CTX-M & $9(27 \%)$ & $2(28 \%)$ & $11(27.5 \%)$ \\
2 & TEM & $10(30 \%)$ & $1(14 \%)$ & $11(27.5 \%)$ \\
3 & CTX-M-1 & $8(24 \%)$ & $1(14 \%)$ & $9(22.5)$ \\
4 & CTX-M-4 & $3(9 \%)$ & $1(14 \%)$ & $4(10 \%)$ \\
5 & NDM-1 & $4 / 20(20 \%)$ & 0 & $4(20 \%)$ \\
\hline
\end{tabular}

showed amplification for CTX-M gene only. Overall, two $(28 \%)$ isolates showed amplification for $C T X-M$ gene.Primers were used to sub-classify four CTX-M genes. Only CTX-M -1 and CTX-M -4 were detected. No amplification was obtained for CTX-M-2 and CTX-M-3 genes (Table-III). When the CTX-M positive isolates were checked for CTX-M type 1, eight (24\%) isolates of E. coli and one (14\%) isolate of $K$. pneumoniae showed amplification. Three (9\%) isolates of E. coli and one (14\%) isolate of K. pneumoniae showed amplification for CTX-M type 4. All the ESBL producing isolates showing resistance to carbapenem were checked for the carbapenemase production. Four out of 20 (20\%) isolates showed amplification for NDM-1gene (Table-III).

\section{DISCUSSION}

ESBL producers are a great health threat especially in nosocomial settings due to their exceptional ability to hydrolyze the latest generations of $\beta$-lactam antibiotics. Their prevalence has increased in an alarming manner in recent years in both hospital and community settings.

The objective of the current study was to find the prevalence of relevant ESBL and carbapenemase producing genes in E. coli and K. pneumoniae isolates from a major hospital in Faisalabad and to check phenotypic susceptibility of all ESBL positive isolates to carbapenems (imipenem, meropenem). This was necessitated because phenotypic effect may be caused by multiple genes although it was not possible to include all reported genes in this study.

In this study, 40 ESBL producing E. coli $(n=33)$ and Klebsiella pneumoniae $(\mathrm{n}=7)$ were included. According to our results, six (18\%) isolates of $E$. coli harbored both CTX-M and TEM genes; three $(9 \%)$ were positive for CTX-M gene alone and four $(12 \%)$ for TEM gene alone. In total, nine $(27 \%)$ isolates were positive for $C T X-M$ gene and 10 (30\%) isolates were positive for TEM gene. This finding is in agreement with the report of Coli and coworkers which showed presence of these genes in $35 \%$ isolates. $^{12}$

Of the 7 ESBL producing K. pneumoniae, two $(28 \%)$ isolates were positive for CTX-M gene and one (14\%) isolate for TEM gene. This finding is in disagreement with a study in a hospital of Iran where $92 \%$ and $76 \%$ isolates were positive for CTX-M and TEM genes respectively. ${ }^{13}$

We further segregated the CTX-M positive isolates (E. coli 9/33 [27\%] and K. pneumoniae 2/7 [28\%]) to check the presence of various subclasses of CTX-M genes. Consensus primers, which recognize variant genes of blaCTX-Ms to date, were used. Only CTX-M-1 and CTX-M-4 genes were detected. $C T X-M-2$ and $C T X-M-3$ genes were not detected in any isolate.

Our finding of $24 \%$ isolates of E. coli testing positive for CTX-M-1 $\beta$-lactamase gene is in strong agreement with a previous study in which $25 \% \mathrm{E}$. 
coli were reported to be CTX-M-1 positive. ${ }^{14}$ Among our eight E.coli isolates, three were also carrying CTX-M-4 gene.

We were able to detect one isolate of $K$. pneumoniae which was positive for both $C T X-M-1$ and CTX-M-4 genes. This is a unique finding as some other researchers were not able to detect CTX-M-4 gene in any of K. pneumoniae isolates. ${ }^{15,16}$ The possible reasons might be the low level of resistance in community and nosocomial infections due to different geographical locations.

On the other hand, we were not able to detect CTX-M-2 and CTX-M-3 genes in any of our isolates. It is in contrast to the findings of some other researchers who were able to detect these genes in $5-10 \%$ isolates which might be from community settings while in our study all of the samples were obtained from tertiary healthcare hospital. ${ }^{17}$

Sixty percent of the 40 ESBL-producing isolates in this report were negative by PCR for blaCTX-M. This may be due to other/yet unexplored CTXMs. Another reason may be the presence of mutated blaCTX-Ms. It highlights the importance of the synergistic use of phenotypic methods and molecular methods for comprehensive understanding the resistance patterns and mechanisms.

We observed that approximately $50 \%$ of $E$. coli and $57 \%$ of K. pneumoniae isolates were resistant to both carbapenems, i.e., imipenem and meropenem phenotypically. This observation is in agreement with a report where resistance pattern was $60 \%$ and $70 \%$ against imipenem and meropenem respectively in $E$. coli isolates. ${ }^{18}$ However, our findings are not in agreement with a recent study in which only $30 \%$ isolates of Klebsiella pneumoniae were resistant to both imipenem and meropenem. ${ }^{19}$ The reason may be that we studied only isolates known to produce ESBLs.

According to a report on carbapenemresistant Enterobacteriaceae isolates, NDM-1 was detected in $75 \%(27 / 36)$ and $66 \%(10 / 15)$ K. pneumoniae and E. coli respectively. ${ }^{20}$ In our study, 50\% ESBL producers (all E. coli) were carbapenemase producers and among them only four isolates $(20 \%)$ were positive for NDM1gene. No K. pneumoniae isolate was found positive. The reason may be that the carbapenem resistance may be due to some other recently discovered genes including $O X A-48, K P C-3$ and VIM-1 genes..$^{21-24}$

\section{CONCLUSION}

The co-existence of ESBL encoding genes (CTX-M-1 and CTX-M-4 group) in both E. coli and K. pneumoniae is indicative of rising threat posed by ESBL producing E. coli and K. pneumoniae. Moreover, the co-existence of ESBL and MBL in the same isolate indicated complete resistance towards all $\beta$-lactam antimicrobials especially the carbapenems which were considered the last wall of defense against these pathogens.

Grant Support \& Financial Disclosures: HEC Project 7664: Molecular characterization of ESBL and carbepenemase producing isolates from Pakistan.

Conflict of Interest: None.

\section{REFERENCES}

1. Moayednia R, Shokri D, Mobasherizadeh S, Baradaran A, Fatemi SM, Merrikhi A. Frequency assessment of $\beta$-lactamase enzymes in Escherichia coli and Klebsiella isolates in patients with urinary tract infection. J Res Med Sci. 2014;19:S41-S45.

2. Medeiros AA. Recent increases in resistance: mechanisms and organisms. Evolution and Dissemination of $\beta$-Lactamases Accelerated by Generations of $\beta$-Lactam Antibiotics. Clin Infect Dis. 1997;24:19-45.

3. Paterson DL, Bonomo RA. Clinical Update ExtendedSpectrum Beta-Lactamases: A Clinical Update. Clin Microbiol Rev. 2005;18:657-686.

4. Ojer-Usoz E, Gonzalez D, Garcia-Jalon I, Vitas AI. High dissemination of extended-spectrum $\beta$-lactamaseproducing Enterobacteriaceae in effluents from wastewater treatment plants. Water Res. 2014;56:37-47. doi: 10.1016/j. watres.2014.02.041

5. Zurfluh K, Hachler H, Nuesch-Inderbinen M, Stephan R. Characteristics of Extended-Spectrum $\beta$-Lactamase and Carbapenemase-Producing Enterobacteriaceae Isolates from Rivers and Lakes in Switzerland. Appl Environ Microbiol. 2013;79:3021-3026. doi: 10.1128/AEM.00054-13

6. Rubtsova MY, Ulyashova MM, Pobolelova YI, Presnova GV, Egorov AM. Biochip for the Simultaneous Identification of Beta-Lactamase and Carbapenemase Genes Conferring Bacterial Resistance to Beta-Lactam Antibiotics. Appl. Biochem. Microbiol. 2020;56:130-140. doi: 10.1134/S000368382002012X

7. Baroud M, Dandache I, Araj GF, Wakim R, Kanj S, Kanafani Z, et al. Underlying mechanisms of carbapenem resistance in extended-spectrum $\beta$-lactamase-producing Klebsiella pneumoniae and Escherichia coli isolates at a tertiary care centre in Lebanon: Role of OXA-48 and NDM1 carbapenemases. Int J Antimicrob Agents. 2013;41:75-79. doi: 10.1016/j.ijantimicag.2012.08.010

8. Paterson DL. Resistance in gram-negative bacteria: Enterobacteriaceae. Am J Infect Control. 2006;34:20-28. doi: 10.1016/j.amjmed.2006.03.013

9. Habeeb MA, Sarwar Y, Ali A, Salman M, Haque A. Rapid emergence of ESBL producers in E. coli causing urinary and wound infections in Pakistan. Pak J Med Sci. 2013;29:540544. doi: $10.12669 /$ pjms.292.3144 
10. Bashir S, Haque A, Sarwar Y, Ali A, Anwar MI. Virulence profile of different phylogenetic groups of locally isolated community acquired uropathogenic E. coli from Faisalabad region of Pakistan. Ann Clin Microbiol Antimicrob. 2012;11:1-6. doi: 10.1186/1476-0711-11-23

11. Cockerill F, Patel J, Alder J, Bradford P, Dudley M, Eliopoulos G. Performance standards for antimicrobial susceptibility testing: twenty-third informational supplement; M100-S23. Wayne, PA: CLSI. 2013.

12. Pitout JD, Hossain A, Hanson ND. Phenotypic and molecular detection of CTX-M- $\beta$-lactamases produced by Escherichia coli and Klebsiella spp. J Clin Microbiol. 2004;42(12):5715-5721. doi: 10.1128/JCM.42.12.57155721.2004

13. Maleki N, Tahanasab Z, Mobasherizadeh S, Rezaei A, Faghri J. Prevalence of CTX-M and TEM $\beta$-lactamases in Klebsiella pneumoniae Isolates from Patients with Urinary Tract Infection, Al-Zahra Hospital, Isfahan, Iran. Adv Biomed Res. 2018;7:10. doi: 10.4103/abr.abr_17_17

14. Brigante G, Luzzaro F, Perilli M, Lombardi G, Colì A, Rossolini GM, et al. Evolution of CTX-M-type $\beta$-lactamases in isolates of Escherichia coli infecting hospital and community patients. Int J Antimicrob Agents. 2005;25:157162. doi: 10.1016/j.ijantimicag.2004.09.013

15. Xiong Z, Zhu D, Wang F, Zhang $Y$, Okamoto R, Inoue M. A Klebsiella pneumoniae producing three kinds of class A $\beta$-lactamases encoded by one single plasmid isolated from a patient in Huashan Hospital, Shanghai, China. Int J Antimicrob Agents. 2004;23:262-267. doi: 10.1016/j. ijantimicag.2003.07.011

16. Hashemizadeh Z, Kalantar-Neyestanaki D, Mansouri S. Clonal relationships, antimicrobial susceptibilities, and molecular characterization of extended-spectrum betalactamase-producing Escherichia coli isolates from urinary tract infections and fecal samples in southeast Iran. Rev Soc Bras Med Trop. 2018;51:44-51. doi: 10.1590/0037-86820080-2017

17. Abrar S, Vajeeha A, Ul-Ain N, Riaz S. Distribution of CTX-M group I and group III $\beta$-lactamases produced by Escherichia coli and Klebsiella pneumoniae in Lahore, Pakistan. Microb Pathog. 2017;103:8-12. doi: 10.1016/j. micpath.2016.12.004. doi: 10.1016/j.micpath.2016.12.004

18. Chang YT, Siu LK, Wang JT, Wu TL, Chen YH, Chuang YC, et al. Resistance mechanisms and molecular epidemiology of carbapenem-nonsusceptible Escherichia coli in Taiwan, 2012-2015. Infect Drug Resist. 2019;12:2113-2123. doi: 10.2147/IDR.S208231
19. Kakian F, Zamzad B, Gholipour A, Zamanzad K. Determination of antibiotic resistance and minimum inhibitory concentration of meropenem and imipenem growth in Klebsiella strains isolated from urinary tract infection in Shahrekord educational hospitals. J Shahrekord Univ Med Sci. 2019;21:80-85. doi: 10.34172/ jsums.2019.14

20. Nagaraj S, Chandran SP, Shamanna P, MacAden R. Carbapenem resistance among Escherichia coli and Klebsiella pneumoniae in a tertiary care hospital in south India. Indian J Med Microbiol. 2012;30:93-95. doi: 10.4103/0255-0857.93054

21. Cristina DA, Lopez-Fresnena N, Rincon Carlavilla AL, Hernandez Garcia M, Ruiz-Garbajosa P, Aranaz-Andres $\mathrm{JM}$, et al. Local prevalence of extended-spectrum betalactamase (ESBL) producing Enterobacteriaceae intestinal carriers at admission and co-expression of ESBL and OXA48 carbapenemase in Klebsiella pneumoniae: A prevalence survey in a Spanish University Hospital. BMJ Open. 2019;9:1-6. doi: 10.1136/bmjopen-2018-024879

22. Ojdana D, Sacha P, Wieczorek P, Czaban S, Michalska A, Jaworowska J, et al. The Occurrence of bla CTX-M, bla SHV, and bla TEM Genes in Extended-Spectrum $\beta$ -Lactamase-Positive Strains of Klebsiella pneumoniae, Escherichia coli, and Proteus mirabilis in Poland. Int J Antibiot. 2014;2014:1-7.

23. Bhattacharjee A, Sen MR, Anupurba S, Prakash P, Nath G. Detection of OXA-2 group extended-spectrum- $\beta$ lactamase-producing clinical isolates of Escherichia coli from India [2]. J Antimicrob Chemother. 2007;60:703-704. doi: 10.1093/jac/dkm267

24. Mushtaq S, Irfan S, Sarma JB, Doumith M, Pike R, Pitout $\mathrm{J}$, et al. Phylogenetic diversity of Escherichia coli strains producing NDM-type carbapenemases. J Antimicrob Chemother. 2011;66(9):2002-2005. doi: 10.1093/jac/dkr226

\section{Authors' Contribution:}
SF: Molecular Biology Experiments.
AA: Sample collection.
AB: Microbiology Experiments.
SA: Sample revival and verification.
AH: Concept and overall supervision. 